\title{
On the Conditions that a given Straight Line may be a Normal to the Quadric Surface
}

$$
(a, b, c, d, f, g, h, u, v, w)(x, y, z, 1)^{2}=0 \text {. }
$$

By R. H. Pinkerton, M.A.

Let the straight line be defined by the coordinates $(a, \beta, \gamma)$ of a point on it and by its direction cosines $l, m, n$. It may be referred to as the line $(\alpha, \beta, \gamma, l, m, n)$. Write, for shortness, the equation to the quadric surface in the form $F(x, y, z)=0$.

The line $(a, \beta, \gamma, l, m, n)$ will be a normal to the quadric if it is perpendicular to either of the tangent planes to the quadric at the points where it cuts the quadric. The equation to this pair of tangent planes may be found as follows :

The line $(\alpha, \beta, \gamma, l, m, n)$ will cut the quadric in two points $\left(x^{\prime}, y^{\prime}, z^{\prime}\right)$, whose distances, $r$, from $(a, \beta, \gamma)$ are the roots of the equation

$$
\mathbf{F}(a+l r, \beta+m r, \gamma+m r)=0,
$$

that is, of the equation

$$
r^{2}(l \mathrm{~L}+m \mathrm{M}+n \mathrm{~N})+2 r(l \mathrm{P}+m \mathrm{Q}+n \mathrm{R})+\mathrm{F}(\alpha, \beta, \gamma,)=0 \quad \ldots
$$

in which

$$
2 \mathrm{~L}=\frac{d \mathrm{~F}(l, m, n)}{d l}-2 \imath, 2 \mathrm{M}, 2 \mathrm{~N}=\text { etc. }
$$

and

$$
2 \mathrm{P}=\frac{d \mathrm{~F}(\alpha, \beta, \gamma)}{d a}, \quad 2 \mathrm{Q}, 2 \mathrm{R}=\text { etc. }
$$

The equation to the tangent plane to the quadric at the point $\left(x^{\prime}, y^{\prime}, z^{\prime}\right)$ is

$$
\begin{aligned}
x\left(a x^{\prime}+h y^{\prime}+g z^{\prime}+u\right) & +y\left(h x^{\prime}+l y^{\prime}+f z^{\prime}+v\right) \\
& \left.+z\left(g x^{\prime}+f y^{\prime}+c z^{\prime}+w\right)+u x^{\prime}+v y^{\prime}+v z^{\prime}+d\right)=0,
\end{aligned}
$$

which, on writing $a+l r, \beta+m r, \gamma+n r$ for $x^{\prime}, y^{\prime}, z^{\prime}$, becomes

$$
r(x \mathrm{~L}+y \mathrm{M}+z \mathrm{~N}+\mathrm{K})+(x \mathrm{P}+y \mathrm{Q}+z \mathrm{R}+\mathrm{S})=0 \quad \ldots
$$

in which

$$
\mathbf{K}=u l+v m+v n
$$

and

$$
\mathrm{S}=u \alpha+v \beta+w \gamma+d \text {. }
$$


If we now eliminate $r$ between the equations (1) and (2), we shall obtain the required equation to the pair of tangent planes in the form

$$
\begin{gathered}
(x \mathbf{P}+y \mathrm{Q}+z \mathrm{R}+\mathrm{S})^{2}(l \mathrm{~L}+m \mathrm{M}+n \mathrm{~N}) \\
-2(x \mathrm{P}+y \mathrm{Q}+z \mathrm{R}+\mathrm{S})(x \mathrm{~L}+y \mathrm{M}+z \mathrm{~N}+\mathrm{K})(l \mathrm{P}+m \mathrm{Q}+n \mathrm{R}) \\
+\mathrm{F}(\alpha, \beta, \gamma) \cdot(x \mathrm{~L}+y \mathrm{M}+z \mathrm{~N}+\mathrm{K})^{2}=0 .
\end{gathered}
$$

On multiplying this equation by $l \mathrm{~L}+m \mathrm{M}+n \mathrm{~N}$, it will appear that it is equivalent to

$$
\begin{aligned}
& {[(x \mathrm{P}+y \mathrm{Q}+z \mathrm{R}+\mathrm{S})(l \mathrm{~L}+m \mathrm{M}+n \mathrm{~N})} \\
& -(x \mathrm{~L}+y \mathrm{M}+z \mathrm{~N}+\mathrm{K})(l \mathrm{P}+m \mathrm{Q}+n \mathrm{R})]^{2} \\
& =(x \mathrm{~L}+y \mathrm{M}+z \mathrm{~N}+\mathrm{K})^{2}\left[(l \mathrm{P}+m \mathrm{Q}+n \mathrm{R})^{2}\right. \\
& -\mathrm{F}(\alpha, \beta, \gamma) \cdot(l \mathrm{~L}+m \mathrm{M}+n \mathrm{~N})],
\end{aligned}
$$

a form which clearly indicates two planes whose line of intersection is given by the equations

$$
\begin{aligned}
& x \mathrm{P}+y \mathrm{Q}+z \mathrm{R}+\mathrm{S}=0 \\
& x \mathrm{~L}+y \mathrm{M}+z \mathrm{~N}+\mathrm{K}=0 .
\end{aligned}
$$

In the equation just found write

$$
\begin{aligned}
& \mathrm{U} \text { for } l \mathrm{~L}+m \mathrm{M}+n \mathrm{~N}, \\
& \mathrm{~V} \text { for } l \mathrm{P}+m \mathrm{Q}+n \mathrm{R},
\end{aligned}
$$

and

$$
\rho^{2} \text { for }(l \mathrm{P}+m \mathrm{Q}+n \mathrm{R})^{2}-\mathrm{F}(\alpha, \beta, \gamma) \cdot(l \mathrm{~L}+m \mathrm{M}+n \mathrm{~N}) \text {. }
$$

It follows from equation (1) that $\rho^{2}=0$ is the condition that the line $(\alpha, \beta, \gamma, l, m, n)$ may be a tangent line to the quadric, and that $\rho$ is real or imaginary according as the line does or does not cut the quadric in real points.

On making the substitutions indicated we get as the equations to the tangent planes

$$
\begin{array}{r}
(x P+y \mathrm{Q}+z \mathrm{R}+\mathrm{S}) \mathrm{U}-(x \mathrm{~L}+y \mathrm{M}+z \mathrm{~N}+\mathrm{K}) \mathrm{V} \\
\quad= \pm(x \mathrm{~L}+y \mathrm{M}+z \mathrm{~N}+\mathrm{K}) \rho \\
\text { or } \quad \begin{aligned}
x(\mathrm{PU}-\mathrm{LV} \pm \mathrm{L} \rho)+y(\mathrm{QU}-\mathrm{MV} \pm \mathrm{M} \rho) \\
+z(\mathrm{RU}-\mathrm{NV} \pm \mathrm{N} \rho)+(\mathrm{SU}-\mathrm{KV} \pm \mathrm{K} \rho)=0
\end{aligned}
\end{array}
$$

in which the signs in the ambiguities are to be taken all + or all - . 
Now the line $(\alpha, \beta, \gamma, l, m, n)$ is a normal to the quadric if it is perpendicular to either of these planes. Hence the line will be a normal if one of the two following sets of conditions is fulfilled:

$$
\begin{aligned}
& (\mathrm{PU}-\mathrm{LV}+\mathrm{L} \rho) / l=(\mathrm{QU}-\mathrm{MV}+\mathrm{M} \rho) / m=(\mathrm{RU}-\mathrm{NV}+\mathrm{N} \rho) / n \\
& (\mathrm{PU}-\mathrm{LV}-\mathrm{L} \rho) / l=(\mathrm{QU}-\mathrm{MV}-\mathrm{M} \rho) / m=(\mathrm{RU}-\mathrm{NV}-\mathrm{N} \rho) / n .
\end{aligned}
$$

It may be noticed that if both sets of conditions are fulfilled, the line is an axis of the quadric, for the line $(\alpha, \beta, \gamma, l, m, n)$ is then perpendicular to both of the tangent planes at the points where it meets the surface. Hence we arrive at the known conditions that the line $(\alpha, \beta, \gamma, l, m, n)$ may be an axis, viz.,

and

$$
\mathrm{L} / l=\mathbf{M} / m=\mathrm{N} / n,
$$

$$
\mathrm{P} / l=\mathrm{Q} / m=\mathrm{R} / n \text {. }
$$

